\title{
Some recent progress in classical general relativity
}

Felix Finster

Max Planck Institute MIS, Inselstr. 22-26, 04103 Leipzig, Germany

Joel Smoller

Mathematics Department, The University of Michigan, Ann Arbor, Michigan 48109

Shing-Tung Yau

Mathematics Department, Harvard University, Cambridge, Massachusetts 02138

(Received 30 September 1999; accepted for publication 18 February 2000)

In this short survey paper, we shall discuss certain recent results in classical gravity. Our main attention will be restricted to two topics in which we have been involved; the positive mass conjecture and its extensions to the case with horizons, including the Penrose conjecture (Part I), and the interaction of gravity with other force fields and quantum-mechanical particles (Part II). (C) 2000 American Institute of Physics. [S0022-2488(00)00606-X]

\section{POSITIVE MASS CONJECTURE AND RELATED TOPICS}

One of the most difficult problems in classical relativity is to understand how and when singularities form. In the 1960's, Hawking and Penrose proved that the existence of a closed trapped surface in an asymptotically flat spacelike hypersurface gives rise to a singularity in space-time. However, no proof based on pure partial differential equation arguments was found, and many questions remain unanswered.

Given an initial data set $\left(g_{i j}, p_{i j}\right)$ on a three-dimensional manifold so that $g_{i j}$ is asymptotically Euclidean and $p_{i j}$ (the induced second fundamental form in an embedding) falls off asymptotically, it is interesting to ask the following questions:

(1) When will such an initial data set contain a closed trapped surface? If so, how to locate it?

(2) If the initial data contains no closed trapped surface, how to tell whether such a surface will appear at a later time under the evolution of Einstein's equations?

(3) If we assume that the trace of $p_{i j}$ is zero, will a singularity occur without the existence of a closed trapped surface?

(4) If a singularity does occur, what is the structure of the null geodesics in a neighborhood of the singularity, and what is the structure of the curvature tensor in this neighborhood? What is the criterium on the initial data set for the curvature to blow up at the singularity?

(5) Can one define physically relevant local (or quasilocal) quantities such as mass and angular momentum to describe regions in a strongly gravitationally interacting space-time? For example, when two bodies interact, what is the binding energy and what is the mass of the resulting configuration? How can one estimate the gravitational radiation for strongly interacting bodies? How can one justify the linearized theory of gravitational radiation?

For all the above questions related to singularity formation, one usually studies only generic initial data. However, it has been a difficult problem in nonlinear partial differential equations to understand how to perturb away the singularity.

For all these questions, it would be good if the known class of spherically symmetric solutions of the Einstein equations were rich. Except for the Schwarzschild case, such solutions cannot be vacuum solutions. Hence to consider these questions, one is forced to couple gravity to other matter fields. For the case of a massless scalar field, Christodoulou ${ }^{1-4}$ has studied the question of the formation of singularities quite extensively. If a singularity exists, it is located at the origin. 
While much is known in this case, the details of how the singularity forms is still poorly understood. (When naked singularities form, one would like to know the behavior of null geodesics.) Based on numerical studies, Choptuik ${ }^{5}$ has found the new phenomena that, for a one-parameter family of initial data, the mass function exhibits some critical phenomena similar to those which occur in statistical mechanics, at the time when a black hole forms. However, a detailed theoretical study is lacking. An interesting consequence of the above study is that after gravitational radiation, the space-time is either time-asymptotic to the flat space-time or to the Schwarzschild spacetime. This raises an interesting question when we couple gravity to a Yang-Mills field or to Dirac spinors; What is the possible asymptotic state of spherically symmetric initial data? Would those stable coupled solutions found by us (cf. Sec. II below) be the only possible states? When we want to extend the spherically symmetric case to an axisymmetric geometry, the space-time is far more complicated. While it is clear that angular momentum may be used to make many configurations stable, the number of degrees of freedom is large and it is difficult to find solutions of gravity coupled to other fields. (For stationary black holes with a vacuum background, it has to be the Kerr solution.) It is still not known whether one can find multiblack holes which can be stabilized by the addition of angular momentum.

Beyond axisymmetric solutions, Bartnik ${ }^{6,7}$ proposed a class of initial data sets which can be foliated by round spheres. Using this ansatz, he was able to parametrize a large set of initial data having zero or nonnegative scalar curvature. (For the initial data set, if it is a maximal slice, the scalar curvature is always non-negative.) According to his numerical study, this ansatz has been very useful in understanding radiation from a single black hole. Perhaps the theoretical study for critical data in this class would be interesting.

Let us now turn to general space-time with no spherical symmetry. We restrict ourselves to asymptotically flat space-times. In this case, we have asymptotic space-time Lorentzian symmetry. Based on this asymptotic symmetry, it is well known that one can define the concept of mass and linear momentum associated to each initial data set (which is invariant under the Lorentzian symmetry at asymptotic infinity ${ }^{8}$ ). About 20 years ago, Schoen-Yau ${ }^{9}$ (subsequently ${ }^{10}$ and others) proved the positive mass conjecture which says that the total (mass, linear momentum) is a nonspacelike four-vector. The total mass is therefore always non-negative. It is zero only when the space-time is flat.

The positivity of the mass says that the trivial space-time is stable (the dynamic stability among a class of reasonable initial data has recently been demonstrated by Christodoulou and Klainerman ${ }^{11}$ ). However, the nonlinear stability of the Schwarzschild solution is still unknown. Based on the "Cosmic Censorship conjecture," Penrose proposed an inequality relating the total mass of the black hole to the area of the outermost horizon. It says that among all initial data sets with fixed mass, the time-symmetric Schwarzschild solution initial data set has the largest area for its outermost apparent horizon.

In general, if the initial data set is a maximal slice for the space-time, the scalar curvature of the three-dimensional manifold is non-negative. In such a case, the conjecture of Penrose was recently settled by Huisken and Ilmanen ${ }^{12}$ obtaining the optimal result only under the assumption that the outermost black hole is connected. It was based on an idea of Geroch that the Hawking (quasilocal) mass is monotonic along an evolution of a surface $\Sigma_{t}$ which starts from the hole to the sphere at infinity. The evolution is governed by the requirement that it moves the surfaces along the normal direction and with magnitude minus the inverse of mean curvature. Geroch noticed that for the sphere at infinity, the Hawking mass is simply the total mass of the initial data set, while at the black hole, the Hawking mass is, up to a universal constant, the square root of the area of the black hole. Hence if the flow of the surface $\Sigma_{t}$ exists, the Penrose conjecture would then be proved. Huisken and Ilmanen developed the mathematical framework in which these ideas could be made precise. However, the flow exhibits jump phenomena and much care is needed to assure that the inequality jumps in the right manner. Much more recently, Bray ${ }^{13}$ has been able to improve the result in the case of a nonconnected outermost horizon by a new method, partly relying on the ideas of Schoen-Yau' and certain curvature estimates. ${ }^{14}$

For the proof of the Penrose conjecture, one still must answer the question as to when the 
initial data set is not maximal. It would also be nice to see the corresponding inequality for the Bondi mass (total mass after radiation).

Besides the total mass and linear momentum, an important conserved quantity is angular momentum. This was studied extensively by Ashtekar. ${ }^{15}$ One needs to study the relation between angular momentum and other conserved quantities such as the total mass and linear momentum. It seems reasonable to believe that the total mass should dominate the square of the angular momentum if the initial data set is nonsingular.

To better understand angular momentum, Huisken and $\mathrm{Yau}^{16}$ defined the concept of center of mass of an initial data set. It is Lorentz invariant and, remarkably, under the evolution of Einstein's equations, the velocity of the center of gravity is the linear momentum divided by $2 \mathrm{~m}$, where $m$ is the total mass of the initial data set.

One hopes to study all possible naturally conserved quantities and the relations among these conserved quantities, when the initial data set is nonsingular. It is always interesting to know how radiation effects all those quantities. For an isolated gravitational system, what would be its asymptotic state after radiation? We conjecture that the time-asymptotic state is just the superposition of several known stationary solutions including the charged Kerr black holes and the static coupled solutions found above (e.g., when we are coupling the Einstein equations to the YangMills, Dirac particles, or a real scalar field).

The global behavior of the Einstein system is difficult to study, partially because we do not have (quasi-)local quantities which behave well under time evolution. The Hawking mass is one such example. It is monotonic in some directions. Unfortunately, it is not positive in general. For certain important closed surfaces, which are obtained by minimizing area under a volume constraint, Christodoulou and Yau were able to prove the positivity of the Hawking mass. ${ }^{17}$ However, they assumed that the scalar curvature is non-negative. It would be nice to remove this assumption.

If one considers sufficient conditions for the formation of black holes in a general setting, the best theorem is due to Schoen-Yau. ${ }^{18}$ This says that, by suitably defining the diameter $d(\Omega)$ of a region $\Omega$, then if the matter density in the region $\Omega$ is greater than $d^{-2}$ up to a universal constant, a closed trapped surface can be found. This implies that a black-hole type singularity exists. In this theorem, the existence of black holes results from the condensation only of matter. It would be desirable to include the contribution of gravitation effects. Namely, it is interesting that in the argument by Schoen-Yau, only the lower bound of the first eigenvalue of the operator $-\Delta$ $+\frac{1}{6} R$ is used. In the time symmetric case (i.e., with $p_{i j} \equiv 0$ ), $\frac{1}{2} R$ is the local matter density. It would be nice to see if this method can be extended to the general case, in the sense that the spectrum of some operator can be used to yield a condition for the formation of black holes.

\section{THE INTERACTION OF GRAVITY WITH OTHER FORCE FIELDS AND DIRAC PARTICLES}

According to Einstein's Theory of General Relativity, gravity is described geometrically through Einstein's equations. The understanding of gravity has been driven by the discovery of special solutions of these equations. The most important examples are the Schwarzschild solution, the Kerr-Newman solution, and the Friedmann-Robertson-Walker solution. ${ }^{19}$ Particularly interesting effects are obtained when one couples gravity, as expressed through Einstein's equations, to other fundamental force fields. The simplest such example is the Reissner-Nordström solution resulting from the coupling of gravity to electromagnetism (Maxwell's equations). This solution, like the Schwarzschild solution, has an essential singularity at the origin. The generalization to non-Abelian Yang-Mills fields led to the discovery of Bartnik and McKinnon (BM) (Ref. 20) (see also Refs. 21 and 22) of everywhere regular solutions. This came as a surprise because several results for related systems led to the conjecture that such solutions cannot exist. Indeed, neither the vacuum Einstein equations, nor the pure Yang/Mills equations have nontrivial static, globally defined, regular solutions. ${ }^{23,24}$ The existence of these solutions depends on the coupling of the different force fields, whereby the attractive gravitational force is balanced by the YM repulsive force. But this balance is rather delicate; for example, the BM solutions are unstable with respect 
to small perturbations. ${ }^{25}$ Other interesting solutions of Einstein's equations result from coupling gravity to quantum mechanical matter fields. The case of a complex scalar field was considered by Lee et al. ${ }^{26}$ who found solitonlike solutions modeling (bosonic) stars; see too, Christodoulou ${ }^{2}$ who studied the gravitational collapse of a real massless scalar field.

We report here on recent work (see Refs. 27-30 for details) of a different type of coupling; namely, gravity coupled to both quantum mechanical particles with spin (Dirac particles), and to an electromagnetic field. We first study the resulting Einstein-Dirac-Maxwell (EDM) equations for a static, spherically symmetric system of two fermions in a singlet spinor state. We find stable solitonlike solutions, and we discuss their properties for different values of the electromagnetic coupling constant. We note too that the inclusion of gravity has a regularizing effect on solutions, in the sense that our solutions are more regular than one would expect from a naive analysis of the Feynman diagrams; see Ref. 31. We then study black-hole solutions for these equations (see Refs. 21 and 32), and we find, surprisingly, that under rather weak regularity conditions on the form of the event horizon, the only black-hole solutions of the EDM equations are the ReissnerNordström (RN) solutions. That is, the spinors must vanish identically. Applying this to the gravitational collapse of a "cloud" of relativistic spin- $\frac{1}{2}$-particles to a black hole, our result indicates that the Dirac particles must eventually disappear inside the event horizon. We also show that the Dirac equation has no normalizable, time-periodic solutions in a RN black-hole background. The physical interpretation of this result is that the Dirac particles cannot remain on a periodic orbit around the black hole. This result has recently been extended to an axisymmetric black hole geometry. ${ }^{33}$

In our study of the coupled EDM equations, we employ a special ansatz for the spinors. In this ansatz, we do not assume that the Dirac particles are in a spherically symmetric state; indeed they are allowed to have angular momentum. However, we arrange $(2 j+1)$ of these particles in such a way that the total system is static and spherically symmetric. (In the language of atomic physics, we consider the completely filled shell of states with angular momentum $j$. Classically, this multiparticle system can be thought of as several Dirac particles rotating around a common center such that their angular momentum adds up to zero.) Since the system of fermions is spherically symmetric, we obtain a consistent set of equations if we also assume spherical symmetry for the gravitational and electric fields. We can thus separate out the angular dependence, and the problem then reduces to a system of nonlinear ODEs.

\section{A. The EDM equations}

The general Einstein-Dirac-Maxwell equations are

$$
R_{j}^{i}-\frac{1}{2} R \delta_{j}^{i}=-8 \pi T_{j}^{i}, \quad(G-m) \Psi_{a}=0, \quad \nabla_{k} F^{j k}=4 \pi e \sum_{a} \overline{\Psi_{a}} G^{j} \Psi_{a},
$$

where $T_{j}^{i}$ is the sum of the energy-momentum tensor of the Dirac particles and the Maxwell stress-energy tensor. The $G^{j}$ are the Dirac matrices which are related to the Lorentzian metric via the anticommutation relations,

$$
\left.g^{j k}(x)\right]=\frac{1}{2}\left\{G^{j}(x), G^{k}(x)\right\} \equiv \frac{1}{2}\left(G^{j} G^{k}+G^{k} G^{j}\right)(x) .
$$

$F^{j k}$ denotes the electromagnetic field tensor, and $\Psi_{a}$ are the wave functions of fermions of mass $m$ and charge $e$. The Dirac operator is denoted by $G$, and it depends on both the gravitational and electromagnetic field; for details see Refs. 27 and 28.

We now specialize to the case of static, spherically symmetric solutions of the EDM system (1). In polar coordinates $(t, r, \vartheta, \varphi)$, we write the metric in the form

$$
d s^{2}=\frac{d t^{2}}{T(r)^{2}}-\frac{1}{A(r)} d r^{2}-r^{2}\left(d \vartheta^{2}+\sin ^{2} \vartheta d \varphi^{2}\right)
$$


with positive functions $T$ and $A$. Depending on whether we consider particlelike solutions or black-hole solutions, the region of space-time which we consider is $r>0$, or $r>\bar{r}>0$, respectively; in the latter case, we assume that $r=\bar{r}$ is the event horizon. We always consider solutions for which the metric (2) is asymptotically Minkowskian,

$$
\lim _{r \rightarrow \infty} A(r)=1=\lim _{r \rightarrow \infty} T(r),
$$

and has finite (ADM) mass; i.e.,

$$
\lim _{r \rightarrow \infty} \frac{r}{2}(1-A(r))=\rho<\infty
$$

In the static case, the fermions only generate an electric field, and thus we may assume that the electromagnetic potential $\mathcal{A}$ has the form $\mathcal{A}=(-\phi, \mathbf{0})$, where $\phi=\phi(r)$ is the Coulomb potential.

The Dirac operator $G$ can be written as

$$
\begin{aligned}
G & =i G^{j}(x) \frac{\partial}{\partial x^{j}}+B(x) \\
& =i T \gamma^{0}\left(\frac{\partial}{\partial t}-i e \phi\right)+\gamma^{r}\left(i \sqrt{A} \frac{\partial}{\partial r}+\frac{i}{r}(\sqrt{A}-1)-\frac{i}{2} \sqrt{A} \frac{T^{\prime}}{T}\right)+i \gamma^{\vartheta} \frac{\partial}{\partial \vartheta}+i \gamma^{\varphi} \frac{\partial}{\partial \varphi},
\end{aligned}
$$

where $\gamma^{t}, \gamma^{r}, \gamma^{\vartheta}$, and $\gamma^{\varphi}$ are the $\gamma$-matrices in polar coordinates, in Minkowski space namely,

$$
\begin{gathered}
\gamma^{t}=\gamma^{0}, \\
\gamma^{r}=\gamma^{1} \cos \vartheta+\gamma^{2} \sin \vartheta \cos \varphi+\gamma^{3} \sin \vartheta \sin \varphi, \\
\gamma^{\vartheta}=\frac{1}{r}\left(-\gamma^{1} \sin \vartheta+\gamma^{2} \cos \vartheta \cos \varphi+\gamma^{3} \cos \vartheta \sin \varphi\right), \\
\gamma^{\varphi}=\frac{1}{r \sin \vartheta}\left(-\gamma^{2} \sin \varphi+\gamma^{3} \cos \varphi\right),
\end{gathered}
$$

where

$$
\gamma^{0}=\left(\begin{array}{cc}
1 & 0 \\
0 & -1
\end{array}\right), \quad \gamma^{i}=\left(\begin{array}{cc}
0 & \sigma^{i} \\
-\sigma^{i} & 0
\end{array}\right), \quad i=1,2,3
$$

and $\sigma^{i}$ denote the Pauli matrices.

In analogy with the central force problem in Minkowski space, ${ }^{34}$ this Dirac operator commutes with (a) the time translation operator $i \partial_{t}$, (b) the total angular momentum operator $J^{2}$, (c) the $z$ component of the total angular momentum $J_{z}$, and (d) with the operator $\gamma^{0} P$, where $P$ is the parity. Since these operators also commute with each other, any solution of the Dirac equation can be written as a linear combination of solutions which are simultaneous eigenstates of these operators. We use this "eigenvector basis" to separate out both the angular and time dependence, and to calculate the total current and energy momentum tensor of the Dirac particles. Using the ansatz in Refs. 27, 28, 29, we can describe the Dirac spinors using two real functions $\alpha$, $\beta$. We arrive at the following system of ordinary differential equations for the five real functions $\alpha, \beta, A$, $T$, and $\phi$ :

$$
\sqrt{A} \alpha^{\prime}= \pm \frac{2 j+1}{2 r} \alpha-((\omega-e \phi) T+m) \beta
$$




$$
\begin{gathered}
\sqrt{A} \beta^{\prime}=((\omega-e \phi) T-m) \alpha+\frac{2 j+1}{2 r} \beta, \\
r A^{\prime}=1-A-2(2 j+1)(\omega-e \phi) T^{2}\left(\alpha^{2}+\beta^{2}\right)-r^{2} A T^{2}\left|\phi^{\prime}\right|^{2}, \\
2 r A \frac{T^{\prime}}{T}=A-1-2(2 j+1)(\omega-e \phi) T^{2}\left(\alpha^{2}+\beta^{2}\right) \pm 2 \frac{(2 j+1)^{2}}{r} T \alpha \beta \\
+2(2 j+1) m T\left(\alpha^{2}-\beta^{2}\right)+r^{2} A T^{2}\left|\phi^{\prime}\right|^{2}, \\
r^{2} A \phi^{\prime \prime}=-(2 j+1) e\left(\alpha^{2}+\beta^{2}\right)-\left(2 r A+r^{2} A \frac{T^{\prime}}{T}+\frac{r^{2}}{2} A^{\prime}\right) \phi^{\prime} .
\end{gathered}
$$

Equations (6) and (7) are the Dirac equations (the \pm signs correspond to the two possible eigenvalues of $\left.\gamma^{0} P\right)$; (8) and (9) are the Einstein equations, while Maxwell's equations reduce to the single equation (10). Here $j=\frac{1}{2}, \frac{3}{2}, \ldots$, the constant $\omega$ enters via the plane wave dependence of the spinors; namely, $\exp (-i \omega t)$, and as for the general equations (1), $m$ and $e$ denote the mass and charge, respectively, of the fermions. We also require that, in addition to (3), (4), the electromagnetic potential vanishes at infinity,

$$
\lim _{r \rightarrow \infty} \phi(r)=0
$$

Since Eqs. (6)-(10) are invariant under the gauge transformations,

$$
\phi(r) \rightarrow \phi(r)+\kappa, \quad \omega \rightarrow \omega+e \kappa, \quad \kappa \in \mathbb{R},
$$

we see that (11) can be fulfilled by a suitable gauge transformation, provided that $\phi$ has a limit at infinity.

In Secs. II A-II D, we shall be concerned with two different types of solutions of Eqs. (6)(10); namely, particlelike solutions (smooth solutions defined for all $r \geqslant 0$ ), and black hole solutions (solutions defined for all $r>\bar{r}>0$, where $A(\bar{r})=0$ and $A(r)>0$ for all $r>\bar{r} ; r=\bar{r}$ is the event horizon). In the first case, we require the following normalization condition on the spinors:

$$
\int_{0}^{\infty}\left(\alpha^{2}+\beta^{2}\right) \frac{T}{\sqrt{A}} d r=1 \quad(\text { particlelike })
$$

while in the second case we require that for all $r_{0}>\bar{r}$,

$$
0<\int_{r_{0}}^{\infty}\left(\alpha^{2}+\beta^{2}\right) \frac{T}{\sqrt{A}} d r<\infty \quad \text { (black holes) }
$$

These conditions are necessary in order that the Dirac spinors define physically meaningful wave functions.

\section{B. Particlelike solutions}

In this section we shall describe our numerical construction of particlelike solutions for Eqs. (6)-(10). For simplicity we shall restrict ourselves to the case $j=1 / 2$. We shall also discuss the stability and properties of the ground state solutions for different values of the electromagnetic coupling constant $(\mathrm{e} / \mathrm{m})^{2}$. We shall show that solutions exist even when the em coupling is so strong that the total interaction is repulsive in the nonrelativistic limit. In addition, for small em 
coupling, $(e / m)^{2}<1$, we shall show that stable particlelike solutions exist for small values of $m$, and using certain topological techniques, we show that this stable solution becomes unstable as $m$ increases.

The construction of particlelike solutions is obtained via a rescaling argument (see Refs. 27 and 28). The idea is to weaken the conditions (2), (11), and (13) to

$$
0 \neq \int_{0}^{\infty}\left(\alpha^{2}+\beta^{2}\right) \frac{T}{\sqrt{A}} d r<\infty, \quad 0 \neq \lim _{r \rightarrow \infty} T(r)<\infty, \lim _{r \rightarrow \infty} \phi(r)<\infty,
$$

and instead set

$$
T(0)=1, \quad \phi(0)=0, \quad m=1 .
$$

This enables us to use a Taylor expansion around $r=0$, and we obtain the following expansions near $r=0$ :

$$
\begin{gathered}
\alpha(r)=\alpha_{1} r+\mathcal{O}\left(r^{2}\right), \quad \beta(r)=\mathcal{O}\left(r^{2}\right), \\
A(r)=1+\mathcal{O}\left(r^{2}\right), \quad T(r)=1+\mathcal{O}\left(r^{2}\right), \quad \phi(r)=\mathcal{O}\left(r^{2}\right) .
\end{gathered}
$$

Solutions to our equations now depend on the three real parameters $e, \omega$, and $\alpha_{1}$. For a given value of these parameters, we can construct initial data at $r=0$, and using the standard Mathematica ODE solver, we "shoot" for numerical solutions of the modified system (6)-(10), (16). By varying $\omega$ (for fixed $e$ and $\alpha_{1}$ ), we can arrange that the spinors $(\alpha, \beta)$ tend to the origin for large $r$, and the conditions (4) and (15) also hold.

Given a solution $(\alpha, \beta, A, T, \phi)$ of this modified system, we consider the scaled functions

$$
\begin{gathered}
\widetilde{\alpha}(r)=\sqrt{\frac{\tau}{\lambda}} \alpha(\lambda r), \widetilde{\beta}(r)=\sqrt{\frac{\tau}{\lambda}} \beta(\lambda r), \\
\widetilde{A}(r)=A(\lambda r), \quad \widetilde{T}(r)=\tau^{-1} T(\lambda r), \quad \widetilde{\phi}(r)=\tau \phi(\lambda r) .
\end{gathered}
$$

By direct computation, these functions satisfy the original Eqs. (6)-(10) and Eqs. (3), (4), and (13), provided that the physical parameters are transformed according to

$$
\widetilde{m}=\lambda m, \quad \widetilde{\omega}=\lambda \tau \omega, \quad \widetilde{e}=\lambda e,
$$

where the scale factors $\lambda$ and $\tau$ are given by

$$
\lambda=\left(4 \pi \int_{0}^{\infty}\left(\alpha^{2}+\beta^{2}\right) \frac{T}{\sqrt{A}} d r\right)^{1 / 2}, \quad \tau=\lim _{r \rightarrow \infty} T(r) .
$$

Finally, condition (11) can be fulfilled by a suitable gauge transformation. Notice that the parameter $(\widetilde{e} / \widetilde{m})^{2}=e^{2}$ is invariant under the above scaling. It is thus convenient to choose $(\widetilde{e} / \widetilde{m})^{2}$ (and not $\widetilde{e}^{2}$ ) as the parameter used to describe the strength of the em coupling. We point out that the above scaling technique is used only to simplify the numerics; for the physical interpretation, however, we must always work with the scaled (tilde) solutions. Since the transformation from the un-tilde to the tilde variables is one-to-one, our scaling method yields all the solutions of the original system. From now on, we shall only consider the scaled solutions, and for simplicity in notation, we shall omit the tilde. 

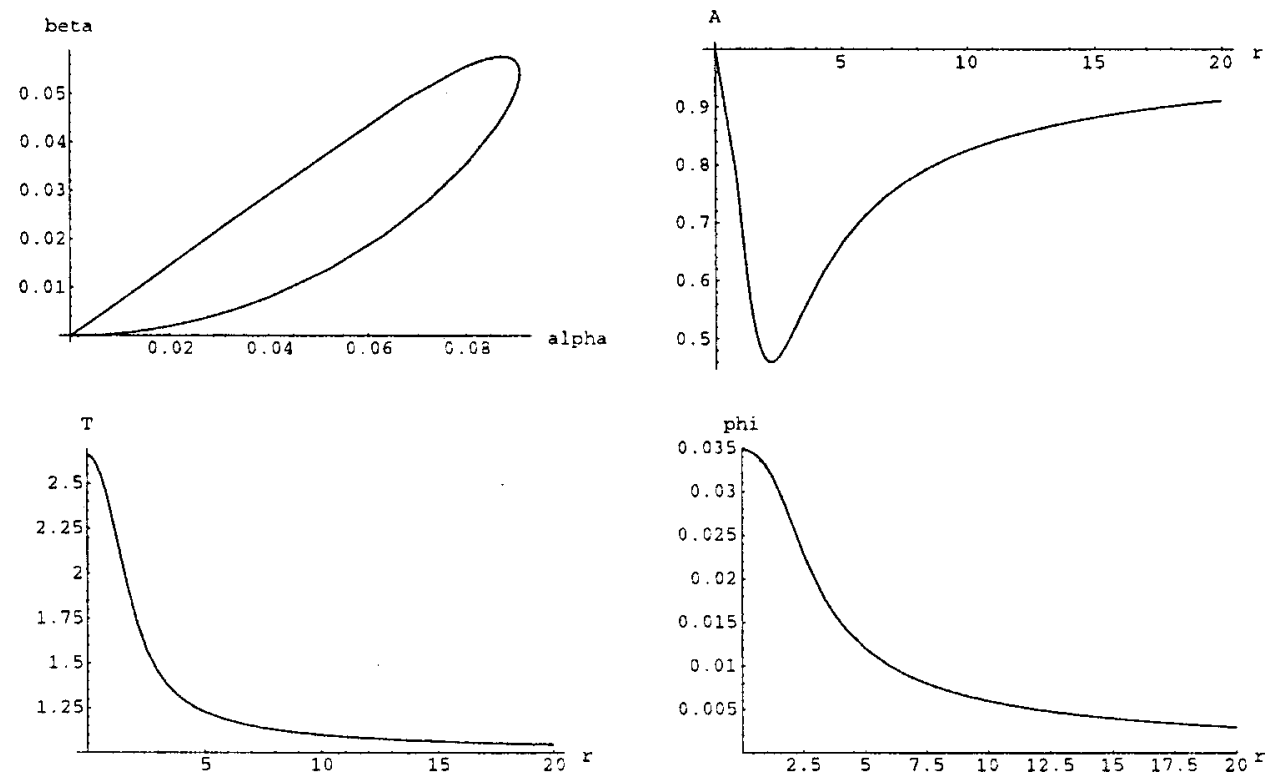

FIG. 1. Solution of the EDM equations for parameter values $(e / m)^{2}=0.7162, m=0.7639, \omega=0.6807, \rho=1.15416\left(\alpha^{\prime}(0)\right.$ $=0.05361)$.

\section{Properties of the particlelike solutions}

We have found solutions having different rotation numbers $n=0,1,2, \ldots$ of the vector $(\alpha, \beta)$. In the nonrelativistic limit, $n$ is the number of zeros of the corresponding Schrödinger wave functions, and thus $n=0$ corresponds to the ground state, $n=1$ to the first excited state, and so on. However due to the nonlinearity of our equations, $n$ no longer has this simple interpretation. For simplicity in what follows, we shall only discuss the $n=0$ solutions. The graphs of a typical such solution is shown in Fig. 1. For each solution, the spinors $(\alpha, \beta)$ decay exponentially to zero at infinity. We interpret this to mean that the fermions have a high probability to be confined to a neighborhood of the origin. In view of this rapid decay of the spinors, our solutions asymptotically go over into the spherically symmetric RN solutions of the Einstein-Maxwell equations, ${ }^{19}$ as $r$ $\rightarrow \infty$. That is, for large $r$,

$$
A(r) \approx T^{-2}(r) \approx 1-\frac{2 \rho}{r}+\frac{(2 e)^{2}}{r^{2}} .
$$

In other words, our solution, for large $r$, looks like the gravitational and electrostatic field generated by a point particle at the origin with mass $m$ and charge $2 e$. Note that in contrast to the RN solution, however, our solutions have no event horizon or singularities. One can understand this from the fact that we consider here quantum mechanical particles, rather than point particles. Therefore the wave functions are delocalized according to the Heisenberg Uncertainty Principle, and so the distributions of matter and charge are also delocalized, thereby preventing the metric from forming singularities. In general, we can parametrize solutions by the rest mass $m$, and the energy $\omega$ of the fermions. In Fig. 2, we plot the binding energy $m-\omega$ vs $m$ for different values of the parameter $(e / m)^{2}$, and we see that $m-\omega$ is always positive, indicating that the fermions are in a bound state. For weak em coupling, $(\mathrm{e} / \mathrm{m})^{2}<1$, the curve is a spiral which starts at the origin. The binding energy decreases for fixed $m$ and increasing $(e / m)^{2}$, since the em repulsion weakens the binding. The mass energy spectrum when $(e / m)^{2} \ll 1$ becomes similar to the case of the Einstein-Dirac equations (without the em interaction); see Ref. 27. We can use linearization techniques to show numerically that for small $m$, if $(e / m)^{2}<1$, the solutions are stable with respect to spherically symmetric perturbations. For larger values of $m$, we can investigate the 


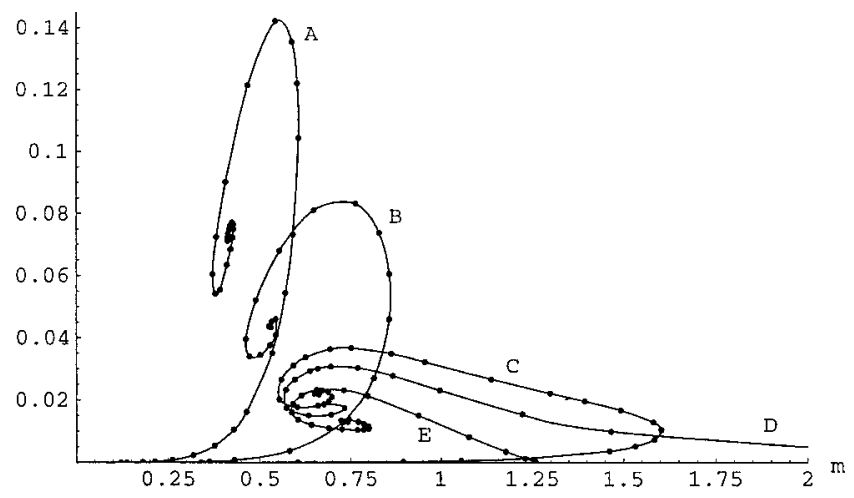

FIG. 2. Binding energy $m-\omega$ of the Fermions for $(e / m)^{2}=0$ (A), 0.7162 (B), 0.9748 (C), 1 (D), and 1.0313 (E).

stability using Conley index theory (see Ref. 35), where $m$ is taken to be the bifurcation parameter. This technique shows that the stability/instability of a solution remains unchanged if $m$ is varied continuously and no bifurcations occur. Moreover, at bifurcation points, the Conley index theory provides a powerful technique to analyze changes of stability. Using this, we find that all solutions on the "lower branch" of the spiral curves A and B of Fig. 2 (i.e., on the curve from the origin up to the maximal value of $m$ ), are stable, and all solutions on the "upper branch" are unstable.

From Fig. 2, we see that this form of the mass energy spectrum changes when $(e / m)^{2} \approx 1$, the regime where, in the classical limit, the electrostatic and gravitational forces balance each other. To better understand this situation, we take the nonrelativistic limit in our EDM equations. To do this, we fix $(e / m)^{2}$, and assume that $e$ and $m$ are small. In this limit, the coupling of the spinors to both the gravitational and $\mathrm{em}$ forces becomes weak; $A, T \approx 1$ and $\phi \approx 0$. The Dirac equations imply that $\omega \approx m$ and $\alpha \gg \beta$. Thus the EDM equations go over to the Schrödinger equation with the Newtonian and Coulomb potentials; namely,

$$
\begin{gathered}
\left(-\frac{1}{2 m} \Delta+e \phi+m V\right) \Psi=E \Psi, \\
-\Delta V=-8 \pi m|\Psi|^{2}, \quad-\Delta \phi=8 \pi e|\Psi|^{2},
\end{gathered}
$$

where $E=\omega-m, \Psi(r)=\alpha(r) / r, V(r)=1-T(r)$, and $\Delta$ is the radial Laplacian on $\mathbb{R}^{3}$. From (19) we see that the Newtonian and Coulomb potentials are multiples of each other; namely, $V$ $=-m / e \phi$. Thus if $(e / m)^{2} \geqslant 1$, the total interaction is repulsive so that the Schrödinger equation (18) has no bound states. It follows that in the limit of small $m$, the EDM equations have no particlelike solutions, if $(\mathrm{e} / \mathrm{m})^{2} \geqslant 1$. This means that the mass-energy curves in Fig. 2 can only start at $m=0$ if $(e / m)<1$. This is confirmed by the numerics (Fig. 2, curves C, D, and E). For $(e / m)^{2}=1$, the curve tends to $m-\omega=0$ as $m \rightarrow \infty$.

If $(e / m)^{2}>1$, Fig. 2 shows that the EDM equations admit solutions only if $m$ is sufficiently large, and smaller than some threshold value where the binding energy of the fermions goes to zero.

We can also consider the total binding energy $\rho-2 m$, where $\rho$ is defined in (4). In Fig. 3, we plot $\rho-2 m$ vs $m$, for various values of $(e / m)^{2}$. If $(e / m)^{2}<1, \rho-2 m$ is negative for the stable solutions, while $\rho-2 m>0$ if $(e / m)^{2}>1$. This indicates that if $(e / m)^{2}>1$, such solutions should be unstable because energy is gained by breaking up the binding.

\section{Nonexistence of black hole solutions}

As we have noted in the last section, particlelike solutions of the EDM equations in a given state (e.g., the ground state) cease to exist if the rest mass $m$ of the fermions exceeds a certain threshold value $m_{s}$. The most natural physical interpretation of this statement is that if $m>m_{s}$, 


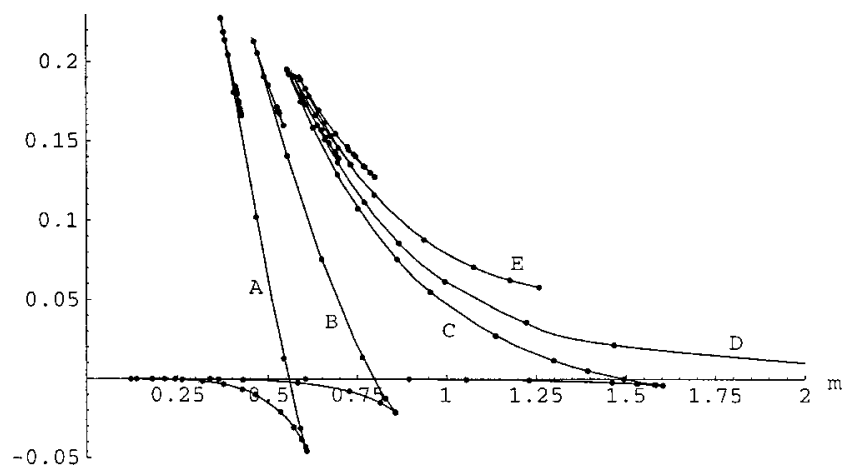

FIG. 3. Total binding energy $\rho-2 m$ for $(e / m)^{2}=0$ (A), 0.7162 (B), 0.9748 (C), 1 (D), and 1.0313 (E).

the gravitational interaction becomes so strong that a black hole would form. This suggests that there should be black hole solutions of the EDM equations for large fermion masses. In this section, we shall show that this intuitive picture of black hole formation is incorrect. In fact, we prove that under weak regularity conditions on the form of the horizon, any black hole solution of the EDM equations must either be the RN solution (in which case the Dirac wave function is identically zero), or the event horizon has the same general form as the extreme RN metric. In the latter case, we show numerically that the Dirac wave functions cannot be normalized. It follows that the EDM system does not admit black hole solutions. Thus the study of black holes in the presence of Dirac spinors leads to unexpected physical effects. If we apply this result to the gravitational collapse of a "cloud" of Dirac particles, our result indicates that the Dirac particles must eventually disappear inside the event horizon.

In order to establish these results, we first recall what is meant by black hole solutions of the EDM equations. These are solutions of Eqs. (6)-(10) defined in the region $r>\bar{r}>0$, which are asymptotically flat (so that (3) holds), and have finite (ADM) mass (so that (4) holds), and satisfy the normalization condition (14). In addition, we assume that $A(r)>0$ for $r>\bar{r}$, and $\lim _{r \backslash \bar{r}} A(r)=0$, while $T(r)>0$ and $\lim _{r \backslash \bar{r}} T(r)=\infty$.

We make the following three assumptions on the regularity of the functions $A, T$, and $\phi$ on the form of the event horizon $r=\bar{r}$ :

(I) The volume element $\sqrt{\left|\operatorname{det} g_{i j}\right|}=r^{2} A^{-1 / 2} T^{-1}$ is smooth and nonzero on the horizon; i.e.,

$$
T^{-2} A^{-1}, T^{2} A \in C^{\infty}([\bar{r}, \infty)) \text {. }
$$

(II) The electromagnetic field tensor is $F_{i j}=\partial_{i} A_{j}-\partial_{j} A_{i}$; we assume that the strength of the em field tensor $F_{i j} F^{i j}=-2\left|\phi^{\prime}\right|^{2} A T^{2}$ is bounded near the horizon. In view of (I), this means that we assume

$$
\left|\phi^{\prime}(r)\right|<c_{1}, \quad \bar{r}<r<\bar{r}+\varepsilon
$$

for some positive constants $c_{1}, \varepsilon>0$.

(III) The function $A(r)$ obeys a power law, i.e.,

$$
A(r)=c(r-\bar{r})^{s}+\mathcal{O}\left((r-\bar{r})^{s+1}\right), \quad r>\bar{r}
$$

for some positive constants $c$ and $s$.

A brief discussion of these assumptions is in order. Thus, if (I) or (II) were violated, then an observer freely falling into a black hole would feel strong forces when crossing the horizon. Assumption (III) is a technical condition which seems sufficiently general to include all physically relevant horizons; for example, $s=1$ corresponds to the Schwarzschild horizon, and $s=2$ corresponds to the extreme RN horizon. However, (III) does not seem to be essential for our nonexistence results, and with more mathematical effort, we believe that it could be weakened or even omitted completely.

Here is the main result in this section.

Theorem 4.1: Any black hole solution of the EDM equations (6)-(10) which satisfies the 
regularity conditions $(I)-(I I I)$ either is a nonextreme $R N$ solution with $\alpha(r) \equiv 0 \equiv \beta(r)$, or $s=2$ and the following expansions are valid near the event horizon $r=\bar{r}$ :

$$
\begin{gathered}
A(r)=A_{0}(r-\bar{r})^{2}+\mathcal{O}\left((r-\bar{r})^{3}\right), \\
T(r)=T_{0}(r-\bar{r})^{-1}+\mathcal{O}\left((r-\bar{r})^{0}\right), \\
\phi(r)=\frac{\omega}{e}+\phi_{0}(r-\bar{r})+\mathcal{O}\left((r-\bar{r})^{2}\right), \\
\alpha(r)=\alpha_{0}(r-\bar{r})^{\kappa}+\mathcal{O}\left((r-\bar{r})^{\kappa+1}\right), \\
\beta(r)=\beta_{0}(r-\bar{r})^{\kappa}+\mathcal{O}\left((r-\bar{r})^{\kappa+1}\right),
\end{gathered}
$$

with positive constants $A_{0}, T_{0}$, and real parameters $\phi_{0}, \alpha_{0}$, and $\beta_{0}$. The exponent $\kappa$ satisfies the constraint

$$
\frac{1}{2}<\kappa=A_{0}^{-1} \sqrt{m^{2}-e^{2} \phi_{0}^{2} T_{0}^{2}+\left(\frac{2 j+1}{2 \bar{r}}\right)^{2}},
$$

and the spinor coefficients $\alpha_{0}$ and $\beta_{0}$ are related by

$$
\alpha_{0}\left(\sqrt{A_{0}} \kappa \pm \frac{2 j+1}{2 \bar{r}}\right)=-\beta_{0}\left(m-e \phi_{0} T_{0}\right)
$$

where " \pm " refers to the two choices of the signs in (6)-(10).

We shall now outline a proof of this result; we first consider the case that the exponent $s$ $<2$ in (20).

Lemma 4.2: Assume that $s<2$ and that $(\alpha \beta, A, T, \phi)$ is a black-hole solution where $(\alpha, \beta) \neq \equiv$. Then there are constants $c, \varepsilon>0$ satisfying

$$
c \leqslant \alpha(r)^{2}+\beta(r)^{2} \leqslant \frac{1}{c}, \quad \bar{r}<r<\bar{r}+\varepsilon .
$$

Proof: According to (6) and (7), we have

$$
\sqrt{A} \frac{d}{d r}\left(\alpha^{2}+\beta^{2}\right)=2\left(\begin{array}{c}
\alpha \\
\beta
\end{array}\right)\left(\begin{array}{cc} 
\pm \frac{2 j+1}{2 r} & -m \\
-m & \mp \frac{2 j+1}{2 r}
\end{array}\right)\left(\begin{array}{l}
\alpha \\
\beta
\end{array}\right) \leqslant\left(4 m^{2}+\frac{(2 j+1)^{2}}{r^{2}}\right)^{1 / 2}\left(\alpha^{2}+\beta^{2}\right) .
$$

The uniqueness theorem for ODEs implies that $\left(\alpha^{2}+\beta^{2}\right)(r)>0$ for all $r, \bar{r}<r<\bar{r}+\varepsilon$, for any $\varepsilon>0$. Dividing (29) by $\sqrt{A}\left(\alpha^{2}+\beta^{2}\right)$ and integrating from $r>\bar{r}$ to $\bar{r}+\varepsilon$ gives

$$
\left|\log \left(\left(\alpha^{2}+\beta^{2}\right)(\bar{r}+\varepsilon)\right)-\log \left(\left(\alpha^{2}+\beta^{2}\right)(r)\right)\right| \leqslant \int_{r}^{\bar{r}+\varepsilon} A^{-1 / 2}(t)\left(4 m^{2}+\frac{(2 j+1)^{2}}{t^{2}}\right)^{1 / 2} d t .
$$

Since $s<2$, (20) implies that $A^{-1 / 2}$ is integrable on $\bar{r} \leqslant r \leqslant \bar{r}+\varepsilon$, so that the integral in (30) is majorized by

$$
\int_{\bar{r}}^{\bar{r}+\varepsilon} A^{-(1 / 2)}(t)\left(4 m^{2}+\frac{(2 j+1)^{2}}{t^{2}}\right)^{1 / 2} d t
$$


and this yields (28).

We can now dispose of the case $0<s<2$; namely, we have

Proposition 4.3: If $0<s<2$, then the only black hole solutions of the systems (6)-(10) are the nonextreme Reissner-Nordström solutions.

Proof: We assume that we have a solution such that $(\alpha, \beta)(r) \equiv \equiv$, and show that this gives a contradiction.

The last lemma implies that the spinors are bounded near $r=\bar{r}$. From (8) and (9), we find

$$
r \frac{d}{d r}\left(A T^{2}\right)=-4(2 j+1)(\omega-e \phi) T^{4}\left(\alpha^{2}+\beta^{2}\right) \pm 2 \frac{(2 j+1)^{2}}{r} T^{3} \alpha \beta+2(2 j+1) m T^{3}\left(\alpha^{2}-\beta^{2}\right) .
$$

Assumption (I) implies that the left-hand side of (31) is regular so the same is true of the right-hand side. Since $T \rightarrow \infty$ as $r \searrow \bar{r}$, we conclude that

$$
\lim _{\bar{r}<r \rightarrow \bar{r}}(\omega-e \phi(r))=0 .
$$

From Maxwell's equation

$$
\phi^{\prime \prime}=-\frac{1}{A} \frac{(2 j+1) e}{r^{2}}\left(\alpha^{2}+\beta^{2}\right)-\frac{1}{r^{2} \sqrt{A} T}\left[r^{2} \sqrt{A} T\right]^{\prime} \phi^{\prime},
$$

we see that (I) implies that the coefficient of $\phi^{\prime}$ is smooth. If $s \geqslant 1, A^{-1}$ is not integrable at $\bar{r}$, so that $\left|\phi^{\prime}\right|$ is unbounded at $\bar{r}$, thereby contradicting (II). Thus $s<1$, and integrating (33) twice and using (32) gives near $r=\bar{r}$ the following expansions:

$$
\phi^{\prime}(r)=c_{1}(r-\bar{r})^{-s+1}+c_{2}+\mathcal{O}\left((r-\bar{r})^{-s+2}\right),
$$

and

$$
\phi(r)=c_{1}(r-\bar{r})^{-s+2}+c_{2}(r-\bar{r})+\frac{\omega}{e}+\mathcal{O}\left((r-\bar{r})^{-s+3}\right) .
$$

Using these in (8), and noting that $A$ and $r^{2} A T^{2}\left|\phi^{\prime}\right|^{2}$ are bounded near $r=\bar{r}$, and that $(\omega-e \phi)$ $=\mathcal{O}(r-\bar{r})$, and $T^{2}\left(\alpha^{2}+\beta^{2}\right) \sim(r-\bar{r})^{-s}, s<1$, we see that the rhs of (8) is bounded near $r=\bar{r}$. On the other hand, the lhs of (8) diverges near $r=\bar{r}$ since $r A^{\prime}(r)=(r-\bar{r})^{-s+1}$; this contradiction completes the proof.

In the case $s \geqslant 2$, we first prove the following two facts (cf. Ref. 29):

$$
\lim _{r \searrow \bar{r}}(r-\bar{r})^{-(s / 2)}\left(\alpha^{2}+\beta^{2}\right)=0
$$

and

$$
\lim _{r \searrow \bar{r}}\left|\phi^{\prime}(r)\right|=\bar{r}^{-1} \lim _{r \searrow \bar{r}} A^{-(1 / 2)} T^{-1}>0
$$

From (35), we find that

$$
(w-e \phi)(r)=c+d(r-\bar{r})+o(r-\bar{r}),
$$

where $d=e / \bar{r} \lim _{r \backslash \bar{r}} A^{-1 / 2} T^{-1}>0$. Thus $(\omega-e \phi) T$ diverges monotonically. From (6) and (7), this implies that $\liminf _{r \backslash \bar{r}}\left(\alpha^{2}+\beta^{2}\right)>0$, thereby contradicting (34). Thus if $s>2$, there are no solutions of (6)-(10). 
Proof of Theorem 4.1: We must only consider the case that $s=2$ and (21), (22) hold. From (34) we see that $\lim _{r \backslash \bar{r}} \alpha^{2}+\beta^{2}=0$, and we can show that $(\omega-e \phi) T$ cannot diverge monotonically near $r=\bar{r}$ (see Ref. 30). But (35) shows that ( $\omega-e \phi)$ has a Taylor expansion near $r=\bar{r}$ with a nonzero linear term. Thus (35) holds, the constant term in the Taylor expansion of $(\omega-e \phi)$ vanishes, and $\lim _{r} \backslash \bar{r}(\omega-e \phi) T=\lambda$, where from (35), $|\lambda|=\bar{r}^{-1} \lim _{r \backslash \bar{r}} A^{-(1 / 2)} T^{-1}>0$. As in Ref. 30 , we may write the Dirac equations in the variable

$$
u(r)=-r-\bar{r} \ln (r-\bar{r})
$$

and apply the stable manifold theorem to conclude that $\alpha$ and $\beta$ satisfy the power laws (24), (25), and (34) yields that $K>\frac{1}{2}$. Using (21)-(25) into (6) and (7) gives

$$
\begin{aligned}
& \sqrt{A_{0}} \kappa \alpha_{0}= \pm \frac{2 j+1}{2 \bar{r}} \alpha_{0}+\left(e \phi_{0} T_{0}-m\right) \beta_{0}, \\
& \sqrt{A_{0}} \kappa \beta_{0}=-\left(e \phi_{0} T_{0}+m\right) \alpha_{0} \mp \frac{2 j+1}{2 \bar{r}} \beta_{0},
\end{aligned}
$$

which are equivalent to (26) and (27). This completes the proof of Theorem 4.1.

Notice that in the case of nonzero spinors $(s=2)$, Theorem 4.1 places severe constraints on the behavior of black hole solutions near the event horizon, in the sense that since $\kappa>\frac{1}{2}$, the spinors decay so fast at $r=\bar{r}$, that both the metric and the $e m$ field behave like the extreme RN solution on the event horizon. Physically speaking, this restriction to the extremal case means that the electric charge of the black hole is so large that the electric repulsion balances the gravitational attraction, and prevents the Dirac particles from "falling into" the black hole. Of course, this is not the physical situation that one expects in the gravitational collapse of, say, a star. However, extreme RN black holes are physically important since they have zero temperature, ${ }^{36}$ and can be considered to be the asymptotic states of black holes emitting Hawking radiation. It is thus interesting to see if the expansions (21)-(25) yield global black hole solutions of the EDM equations.

This question is especially interesting since in the next section we shall show that for an extreme RN background field, spinors satisfying the expansions (24), (25) cannot be normalized. The question thus becomes whether the influence of the spinors on the gravitational and em field can yield black hole solutions with normalized spinors. This is a very difficult question because one must analyze the global behavior of these solutions of the EDM equations. Our numerical investigations show that the answer to the above question is negative; namely solutions either develop a singularity for some $r>\bar{r}$, or the spinors $(\alpha, \beta)$ are not normalizable. We thus conclude that the expansions (21)-(25) do not give normalizable solutions of the EDM equations.

\section{E. Dirac particles in a Reissner-Nordström background}

In this section, we shall consider solutions of the EDM equations where we fix the background metric and em field to be a RN solution. Near a collapsing black hole one might guess that Dirac particles can get into a static or time periodic state. However, we shall show that in contrast to the classical situation, the Dirac equations do not admit any normalizable time-periodic solutions; in particular, they admit no normalizable static solutions. We do not assume any spatial symmetry on the wave functions. This result can be physically interpreted as saying that Dirac particles can either disappear into the black hole of escape to infinity, but they cannot remain on a periodic orbit around the black hole. We note that it is essential for our arguments that the particles have spin. In fact, in the case where the particles do not have spin, the Dirac equation must be replaced by the Klein-Gordon equation, and our arguments fail; cf., Ref. 26.

The RN metric can be written in polar coordinates as 


$$
d s^{2}=\left(1-\frac{2 \rho}{r}+\frac{q^{2}}{r^{2}}\right) d t^{2}-\left(1-\frac{2 \rho}{r}+\frac{q^{2}}{r^{2}}\right)^{-1} d r^{2}-r^{2}\left(d \vartheta^{2}+\sin ^{2} \vartheta d \varphi^{2}\right)
$$

where $\rho$ is the (ADM) mass of the black hole, and $q$ its charge. The em potential is of the form $(-\phi, \mathbf{0})$ with Coulomb potential

$$
\phi(r)=\frac{q}{r} .
$$

In the "nonextremal" case $(q<\rho)$, the metric coefficient $\left(1-(2 \rho / r)+\left(q^{2} / r^{2}\right)\right)$ vanishes twice, and thus there are two horizons $0<r_{0}<r$. If $q=\rho$, the metric is called an extreme ReissnerNordström (ERN) metric and has a single horizon at $r=\rho$. If $q>\rho$, the above metric coefficient is nonvanishing, and so the metric does not describe a black hole; this case will not be considered.

We consider time-periodic solutions, noting that static solutions are a special case. Since the phase of the Dirac wave function $\Psi$ has no physical significance, we define $\Psi$ to be periodic with period $T$ if for some real $\Omega$,

$$
\Psi(t+T, r, \vartheta, \varphi)=e^{-i \Omega T} \Psi(t, r, \vartheta, \varphi) .
$$

Our main theorem in this section is the following:

Theorem 5.1: (i) In a nonextreme RN background, there are no normalizable, time-periodic solutions of the Dirac equation. (ii) In an ERN background, every normalizable, time-periodic solution of the Dirac equation is identically zero in the region $r>\rho$.

We shall begin by deriving conditions which relate the wave function $\Psi$ on both sides of the event horizon. We first consider the case of a nonextreme RN background, and analyze the behavior of $\Psi$ near the event horizon. For this, we begin by studying the behavior of $\Psi$ in a Schwarzchild background metric, and we shall also consider the Dirac equation in different coordinate systems. This is done with the aim of passing to Kruskal coordinates, in order to remove the "Schwarzschild singularity."

The Schwarzschild metric is

$$
d s^{2}=\left(1-\frac{2 \rho}{r}\right) d t^{2}-\left(1-\frac{2 \rho}{r}\right)^{-1} d r^{2}-r^{2}\left(d \vartheta^{2}+\sin ^{2} \vartheta d \varphi^{2}\right)
$$

where $\rho$ is the (ADM) mass, and the event horizon is at $r=2 \rho$. Some straightforward calculations (see Ref. 30), shows that outside the horizon $(r>\rho)$, the Dirac operator can be written as

$$
G_{\mathrm{out}}=\frac{i}{S} \gamma^{t} \frac{\partial}{\partial t}+\gamma^{r}\left(i S \frac{\partial}{\partial r}+\frac{i}{r}(S-1)+\frac{i}{2} S^{\prime}\right)+i \gamma^{\vartheta} \frac{\partial}{\partial \vartheta}+i \gamma^{\varphi} \frac{\partial}{\partial \varphi},
$$

where

$$
S(r)=\left|1-\frac{2 \rho}{r}\right|^{1 / 2}
$$

The normalization integral is considered over the hypersurface $t=$ const; i.e.,

$$
(\Psi \mid \Psi)_{\text {out }}^{t}:=\int_{\mathbb{R}^{3} \backslash B_{2 \rho}}\left(\bar{\Psi} \gamma^{t} \Psi\right)(t, \mathbf{x}) S^{-1} d^{3} x
$$

where $B_{2 \rho}$ denotes the ball of radius $2 \rho$ about the origin, and $\bar{\Psi}=\Psi^{*} \gamma^{0}$ is the adjoint spinor. In the region $r<2 \rho$, the Dirac operator is given by 


$$
G_{\mathrm{in}}=\gamma^{r}\left(\frac{i}{S} \frac{\partial}{\partial t}-\frac{i}{r}\right)-\gamma^{t}\left(i S \frac{\partial}{\partial r}+\frac{i}{r} S+\frac{i}{2} S^{\prime}\right)+i \gamma^{\vartheta} \frac{\partial}{\partial \vartheta}+i \gamma^{\varphi} \frac{\partial}{\partial \varphi}
$$

with corresponding normalization integral

$$
(\Psi \mid \Psi)_{\mathrm{in}}^{t}:=\int_{B_{2 \rho}}\left(\Psi \gamma^{r} \Psi\right)(t, \mathbf{x}) S^{-1} d^{3} x
$$

Our description of spinors in this coordinate system poses certain difficulties. Namely, since the $t$ variable is spacelike inside the horizon, the normalization integral (41) is not definite since the integrand is not positive. Thus we can no longer interpret the integrand as a probability density. Moreover, the Dirac equations corresponding to the operators $G_{\text {in }}$ and $G_{\text {out }}$ describe the wave functions inside and outside the horizon, respectively. But it is not evident how to match the wave functions on the horizon. To handle these issues, we remove the singularity at $r=2 \rho$ by going over to Kruskal coordinates. Recall (see Ref. 19) that Kruskal coordinates $u$ and $v$ are defined by

$$
\begin{aligned}
& u= \begin{cases}\sqrt{\frac{r}{2 \rho}-1} e^{(r / 4 \rho)} \cosh \left(\frac{t}{4 \rho}\right) & \text { for } r>2 \rho \\
\sqrt{1-\frac{r}{2 \rho}} e^{(r / 4 \rho)} \sinh \left(\frac{t}{4 \rho}\right) & \text { for } r<2 \rho\end{cases} \\
& v= \begin{cases}\sqrt{\frac{r}{2 \rho}-1} e^{(r / 4 \rho)} \sinh \left(\frac{t}{4 \rho}\right) & \text { for } r>2 \rho \\
\sqrt{1-\frac{r}{2 \rho}} e^{(r / 4 \rho)} \cosh \left(\frac{t}{4 \rho}\right) & \text { for } \tau<2 \rho\end{cases}
\end{aligned}
$$

The horizon $r=2 \rho$ maps to the origin $u=0=v$, and the singularity $r=0$ maps to the hyperbola $v^{2}-u^{2}=1, v>0$. In Kruskal coordinates, the metric (36) becomes

$$
d s^{2}=f^{-2}\left(d v^{2}-d u^{2}\right)-r^{2}\left(d \vartheta^{2}+\sin ^{2} \vartheta d \varphi^{2}\right),
$$

where $f^{-2}=\left(32 \rho^{3} / r\right) e^{(r / 2 \rho)}$. Taking $v$ and $u$ as time and space variables, respectively, and noting that the metric is regular at the origin, we can extend the Dirac operator smoothly across the origin. A straightforward computation gives the Dirac operator in Kruskal coordinates as

$$
G=\gamma^{t}\left(f i \frac{\partial}{\partial v}+\frac{i}{r} f\left(\partial_{v} r\right)-\frac{i}{2} \partial_{v} f\right)+\gamma^{r}\left(f i \frac{\partial}{\partial v}+\frac{i}{r}\left(f\left(\partial_{u} r\right)-1\right)-\frac{i}{2} \partial_{u} f\right)+i \gamma^{\vartheta} \partial_{\vartheta}+i \gamma^{\varphi} \partial_{\varphi} .
$$

Observe that the Dirac operator is smooth across the event horizon. Moreover, the normalization integrals (40) and (41) on the surface $t=0$ become

$$
(\Psi \mid \Phi)=\int_{\mathcal{H}} \bar{\Psi} G^{j} \Phi \nu_{j} d \mu
$$

where

$$
\mathcal{H}=\{u=0,0 \leqslant v \leqslant 1\} \cup\{v=0, u>0\},
$$

$\nu$ is the normal to $H$ pointing into the region $u>0, v>0$, and $G^{j}$ are the Dirac matrices

$$
G^{v}=f \gamma^{t}, G^{u}=f \gamma^{r}, G^{\vartheta}=\gamma^{\vartheta}, G^{\varphi}=\gamma^{\varphi} .
$$




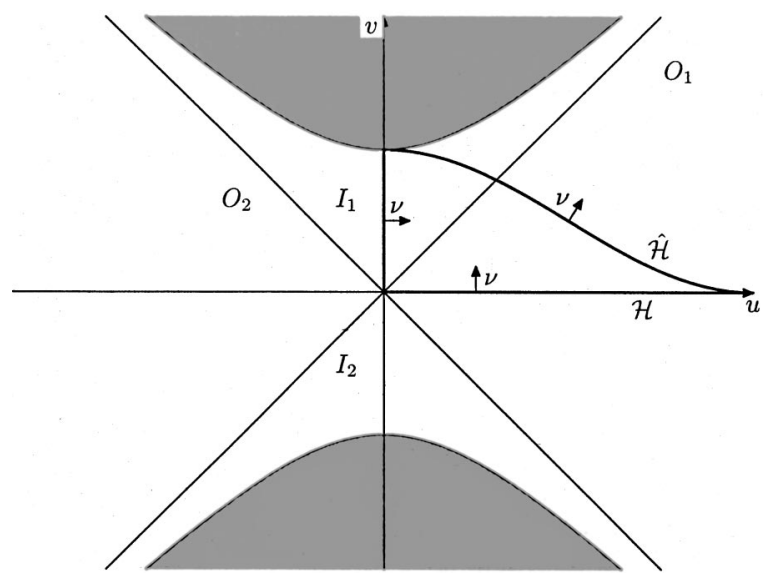

FIG. 4. Kruskal coordinates.

We remark that for smooth solutions of the Dirac equation, one can use current conservation

$$
\nabla \bar{\Psi} G^{j} \Psi=0 .
$$

to continuously deform the hypersurface $\mathcal{H}$ keeping fixed the value of the normalization integral. For example, one can deform $\mathcal{H}$ to $\hat{\mathcal{H}}$ as depicted in Fig. 4, thereby avoiding integrating across the horizon. On the other hand, one must exercise extreme care whenever a solution of the Dirac equation is singular near the origin.

As shown in Ref. 30, the Dirac operator in Kruskal coordinates can be written as

$$
G=U G_{\text {out }} U^{-1}=U G_{\text {in }} U^{-1},
$$

where $U$ is the time-dependent matrix,

$$
U(t)=\cosh \left(\frac{t}{8 \rho}\right) 1+\sinh \left(\frac{t}{8 \rho}\right) \gamma^{t} \gamma^{r},
$$

and the Dirac operators $G_{\text {out }}$ and $G_{\text {in }}$ in Kruskal coordinates are

$$
\begin{aligned}
& G_{\mathrm{out}}=\frac{i}{4 \rho S}\left(u \gamma^{t}+v \gamma^{r}\right) \frac{\partial}{\partial v}+\frac{i}{4 \rho S}\left(v \gamma^{t}+u \gamma^{r}\right) \frac{\partial}{\partial u}+\left(\frac{i}{r}(S-1)+\frac{i}{2} S^{\prime}\right) \gamma^{r}+i \gamma^{\vartheta} \frac{\partial}{\partial \vartheta}+i \gamma^{\varphi} \frac{\partial}{\partial \varphi} \\
& G_{\mathrm{in}}=\frac{i}{4 \rho S}\left(v \gamma^{t}+u \gamma^{r}\right) \frac{\partial}{\partial v}+\frac{i}{4 \rho S}\left(u \gamma^{t}+v \gamma^{r}\right) \frac{\partial}{\partial u}-\left(\frac{i}{r} S+\frac{i}{2} S^{\prime}\right) \gamma^{t}-\frac{i}{r} \gamma^{r}+i \gamma^{\vartheta} \frac{\partial}{\partial \vartheta}+i \gamma^{\varphi} \frac{\partial}{\partial \varphi}
\end{aligned}
$$

It follows that the Dirac operators $G_{\text {out }}$ and $G_{\text {in }}$ can be identified with the Dirac operator $G$ in the region,

$$
\mathcal{R}=\left\{u+v>0, v^{2}-u^{2}<1\right\} .
$$

We next see how solutions of the Dirac equation inside and outside the horizon match on the horizon, $u=0=v$. To do this, we first study the behavior of these solutions on the horizon. Let us first consider static solutions of the Dirac equation, so

$$
\Psi(t, r, \vartheta, \varphi)=e^{-i \omega t} \Psi(r, \vartheta, \varphi) .
$$


We assume that $\Psi$ is a solution of the Dirac equations $\left(G_{\text {in }}-m\right)=0$ and $\left(G_{\text {out }}-m\right)=0$, and that $\Psi$ is smooth on both sides of the horizon $r<2 \rho$ and $r>2 \rho$. Using (46) and (47), we have

$$
\Psi(u, v, \vartheta, \varphi)=U(t) e^{-i \omega t} \Psi(r, \vartheta, \varphi),
$$

where $r$ and $t$ are determined implicitly from $u$ and $v$ in the usual way (see Ref. 19). This implies that $\Psi$ is only defined in $\mathcal{R}$, and solves there the Dirac equation $(G-m) \Psi=0$. Since we are only considering black holes, we demand that $\Psi$ vanishes in the half-plane $u+v<0$; thus we must analyze solutions $\Psi$ of the form

$$
\Psi(u, v, \vartheta, \varphi)=\left\{\begin{array}{l}
U(t) e^{-i \omega t} \Psi(r, \vartheta, \varphi) \quad \text { for } u+v>0, u \neq v \\
0 \quad \text { for } u+v<0
\end{array} .\right.
$$

Such a wave function might be singular along the lines $u= \pm v$, in which case $\Psi$ must satisfy the Dirac equation in a generalized sense. An analysis carried out in Ref. 30 shows that $\Psi$ must satisfy the two matching conditions

$$
\begin{gathered}
\lim _{\varepsilon \rightarrow 0}\left(\gamma^{t}+\gamma^{r}\right)|\varepsilon|^{1 / 4} \Psi(t, 2 \rho+\varepsilon, \vartheta, \varphi)=0 \\
|\varepsilon|^{1 / 4}(\Psi(t, 2 \rho+\varepsilon, \vartheta, \varphi)-\Psi(t, 2 \rho-\varepsilon, \vartheta, \varphi))=o\left(1+|\varepsilon|^{1 / 4} \Psi(t, 2 \rho+\varepsilon, \vartheta, \varphi)\right) \text { as } \quad \varepsilon \rightarrow 0 .
\end{gathered}
$$

Note that since these only depend on the local behavior of $\Psi$ near the horizon, they are also applicable when we are in the case of a nonextreme RN background having event horizon at $r$ $=2 \rho$.

We now consider Dirac particles in a RN background. Since the gravitational and EM background fields are spherically symmetric and time independent, we can separate out the angular and time dependence of the wave functions via spherical harmonics and plane waves in the usual manner and, as shown in Ref. 30, we obtain the following two component Dirac equations: In regions where the $t$-variable is timelike,

$$
S \frac{d}{d r} \Phi_{j k \omega}^{ \pm}=\left[\left(\begin{array}{cc}
0 & -1 \\
1 & 0
\end{array}\right)(\omega-e \phi) \frac{1}{S} \pm\left(\begin{array}{cc}
1 & 0 \\
0 & -1
\end{array}\right) \frac{2 j+1}{2 r}-\left(\begin{array}{ll}
0 & 1 \\
1 & 0
\end{array}\right) m\right] \Phi_{j k \omega}^{ \pm},
$$

and in the regions where $t$ is spacelike,

$$
S \frac{d}{d r} \Phi_{j k \omega}^{ \pm}=\left[\left(\begin{array}{cc}
0 & -1 \\
1 & 0
\end{array}\right)(\omega-e \phi) \frac{1}{S} \pm i\left(\begin{array}{cc}
0 & 1 \\
1 & 0
\end{array}\right) \frac{2 j+1}{2 r}+i\left(\begin{array}{cc}
1 & 0 \\
0 & -1
\end{array}\right) m\right] \Phi_{j k \omega}^{ \pm} .
$$

In these equations,

$$
S(r)=\left|1-\frac{2 \rho}{r}+\frac{q^{2}}{r^{2}}\right|^{1 / 2}
$$

$j=\frac{1}{2}, \frac{3}{2}, \ldots, k=-j,-j+1, \ldots, j$, and the \pm signs correspond as before to the two eigenvalues of the operator $\gamma^{0} P$ (cf. Sec. II). Here we have chosen for the Dirac wave functions the two ansatz'

$$
\begin{aligned}
& \Psi_{j k \omega}^{+}=e^{-i \omega t} \frac{S^{-1 / 2}}{r}\left(\begin{array}{c}
\chi_{j-1 / 2}^{k} \Phi_{j k \omega 1}^{+}(r) \\
i \chi_{j+1 / 2}^{k} \Phi_{j k \omega 2}^{+}(r)
\end{array}\right), \\
& \Psi_{j k \omega}^{-}=e^{-i \omega t} \frac{S^{-1 / 2}}{r}\left(\begin{array}{c}
\chi_{j+1 / 2}^{k} \Phi_{j k \omega 1}^{-}(r) \\
i \chi_{j-1 / 2}^{k} \Phi_{j k \omega 2}^{-}(r)
\end{array}\right),
\end{aligned}
$$

with 2-spinors $\Phi_{j k \omega}^{ \pm}$, and $\chi_{j \pm 1 / 2}^{k}$ are defined by 


$$
\begin{gathered}
\chi_{j-1 / 2}^{k}=\sqrt{\frac{j+k}{2 j}} Y_{j-1 / 2}^{k-1 / 2}\left(\begin{array}{l}
1 \\
0
\end{array}\right)+\sqrt{\frac{j-k}{2 j}} Y_{j-1 / 2}^{k+1 / 2}\left(\begin{array}{l}
0 \\
1
\end{array}\right), \\
\chi_{j+1 / 2}^{k}=\sqrt{\frac{j+1-k}{2 j+2}} Y_{j+1 / 2}^{k-1 / 2}\left(\begin{array}{l}
1 \\
0
\end{array}\right)-\sqrt{\frac{j+1+k}{2 j+2}} Y_{j+1 / 2}^{k+1 / 2}\left(\begin{array}{l}
0 \\
1
\end{array}\right),
\end{gathered}
$$

where $Y_{l}^{k}$ are the usual spherical harmonics, $l=0,1,2, \ldots, k=-l, \ldots, l$.

We shall show that the matching conditions (48), (49) do not yield normalizable, timeperiodic solutions of the Dirac equation. This will be done by showing that, for every nonzero solutions of Dirac's equation, the normalization integral outside and away from the horizons

$$
(\Psi \mid \Psi)^{t}=\int_{\mathbb{R}^{3} \backslash B_{2 r_{1}}} \bar{\Psi} \gamma^{t} \Psi S^{-1} d^{3} x
$$

is infinite for some $t$. Note that for a normalizable wave function, this integral is the probability that the particle lies outside the ball of radius $r_{1}$, and thus cannot exceed 1. So if (57) is infinite, the wave function cannot be normalized.

Now assume that $\Psi$ is a $T$-periodic solution of Dirac's equation. Expanding the periodic function $e^{-i \Omega t} \Psi(t, r, \vartheta, \varphi)$ in a Fourier series, and using the basis (51), (52) yields

$$
\Psi(t, r, \vartheta, \varphi)=\sum_{n, j, k, s} \Psi_{j k \omega(n)}^{s}(t, r, \vartheta, \varphi),
$$

where $s= \pm$, and $\omega(n)=\Omega+2 \pi n / T$. Using the orthonormality of the spinors $\chi_{j \pm 1 / 2}^{k}$, the integral (55) becomes

$$
(\Psi \mid \Psi)^{t}=\int_{\mathbb{R}^{3} \backslash B_{2 r_{1}}} \sum_{n, n} \sum_{j, k, s} \overline{\Psi_{j k \omega(n)}^{s}} \gamma^{t} \Psi_{j k \omega\left(n^{\prime}\right)}^{s} S^{-1} d^{3} x
$$

In order to eliminate the oscillating time dependence of the integrand, we average over one period $(0, T)$ to get

$$
\frac{1}{T} \int_{0}^{T}(\Psi \mid \Psi)^{t} d t=\sum_{n, j, k, s}\left(\Psi_{j k \omega(n)}^{s} \mid \Psi_{j k \omega(n)}^{s}\right)
$$

For a normalizable wave function, this expression is finite, and hence all summands are finite. For all $s, j, k, n$,

$$
\left(\Psi_{j k \omega(n)}^{s} \mid \Psi_{j k \omega(n)}^{s}\right)<\infty .
$$

We shall show that (59) cannot hold for nontrivial solutions of the Dirac equation; for this we begin with

Lemma 5.2: The function $\left|\Phi_{j k \omega}^{ \pm}(r)\right|^{2}$ has finite boundary values on both horizons, and if it is zero on one horizon, then it is identically zero.

Proof: For simplicity, we omit the indices $j, k$, and $\omega$. Choose $\delta, 0<\delta<r_{0}$, and notice that the $t$-direction is timelike on the intervals $\left(\delta, r_{0}\right)$ and $\left(r_{1}, \infty\right)$. In these regions, we can use (50) to obtain

$$
\begin{aligned}
S \frac{d}{d r}\left|\Phi^{ \pm}\right|^{2}(r) & =\left\langle S \frac{d}{d r} \Phi^{ \pm}, \Phi^{ \pm}\right\rangle+\left\langle\Phi^{ \pm}, S \frac{d}{d r} \Phi^{ \pm}\right\rangle \\
& = \pm \frac{2 j+1}{r}\left(\left|\Phi_{1}^{ \pm}\right|^{2}-\left|\Phi_{2}^{ \pm}\right|^{2}\right)-4 m \operatorname{Re}\left(\left(\Phi_{1}^{ \pm}\right)^{*} \Phi_{2}^{ \pm}\right),
\end{aligned}
$$


so that

$$
-c\left|\Phi^{ \pm}\right|^{2} \leqslant S \frac{d}{d r}\left|\Phi^{ \pm}\right|^{2} \leqslant c\left|\Phi^{ \pm}\right|^{2},
$$

where $c=2 m+(2 j+1) / \delta$. Dividing by $\left|\Phi^{ \pm}\right|^{2}$ and integrating gives, for $\delta<r<r^{\prime}<r_{0}$, or $r_{1}$ $<r<r^{\prime}$,

$$
-c \int_{r}^{r^{\prime}} S^{-1} \leqslant\left.\log \left|\Phi^{ \pm}\right|^{2}\right|_{r} ^{r^{\prime}} \leqslant c \int_{r}^{r^{\prime}} S^{-1}
$$

In the region $r_{0}<r<r_{1}$, (51) gives similarly

$$
S \frac{d}{d r}\left|\Phi^{ \pm}\right|^{2}(r)=\left\langle S \frac{d}{d r} \Phi^{ \pm}, \Phi^{ \pm}\right\rangle+\left\langle\Phi^{ \pm}, S \frac{d}{d r} \Phi^{ \pm}\right\rangle=0,
$$

since the square bracket in (51) is an anti-Hermitian matrix. Thus $\left|\Phi^{ \pm}\right|^{2}$ is constant in this region, so (60) trivially holds for $r_{0}<r<r^{\prime}<r_{1}$. Since $S^{-1}$ is integrable on the event horizons, (60) shows that $\left|\Phi^{ \pm}\right|^{2}$ has finite boundary values on each side of the horizon, and these are nonzero unless if $\Phi^{ \pm}$vanishes identically on the corresponding region $\left(\delta, r_{0}\right),\left(r_{0}, r_{1}\right)$, or $\left(r_{1}, \infty\right)$.

We now use (53) and (54) in the matching condition (49) to get for $j=0,1$,

$$
\Phi^{ \pm}\left(r_{j}+\varepsilon\right)-\Phi^{ \pm}\left(r_{j}-\varepsilon\right)=o\left(1+\left|\Phi^{ \pm}\left(r_{j}+\varepsilon\right)\right|\right), \quad \varepsilon \rightarrow 0 .
$$

Since $\left|\Phi^{ \pm}(r)\right|^{2}$ has 2 -sided limits as $r_{j}$, we conclude that these limits must coincide at $r_{j}$; i.e.,

$$
\lim _{0<\varepsilon \rightarrow 0}\left|\Phi^{ \pm}\left(r_{j}+\varepsilon\right)\right|^{2}=\lim _{0<\varepsilon \rightarrow 0}\left|\Phi^{ \pm}\left(r_{j}-\varepsilon\right)\right|^{2}
$$

Using (60) again, we conclude that the wave function vanishes on the entire interval $(\delta, \infty)$, if it is zero on $r_{j}$. This completes the proof since $\delta$ was arbitrary.

The final step is to use current conservation (cf. Ref. 27),

$$
\nabla_{j} \bar{\Psi} G^{j} \Psi=0
$$

to study the decay of $\Phi_{j k \omega(n)}^{s}(r)$ at infinity, and to prove Part (i) of Theorem 5.1.

Theorem 5.3 (radial flux argument): Either $\Psi_{j k \omega}^{s}$ vanishes identically, or the normalization condition (59) is violated.

Proof: For simplicity, we again omit the indices $s, j, k$, and $\omega$. Suppose that $\Psi \neq \equiv$. For $r_{1}$ $<r<R$ and $T>0$, let $V$ be the annulus outside the horizon $r$, given by $V=(0, T) \times\left(B_{2 R} \backslash B_{2 r}\right)$. Using (59), we find

$$
\begin{aligned}
0= & \int_{V} \nabla_{j}\left(\bar{\Psi} G^{j} \Psi\right) \sqrt{|g|} d^{4} x \\
= & \int_{0}^{T} d t r^{2} S(r) \int_{S^{2}}\left(\bar{\Psi} \gamma^{r} \Psi\right)(t, r)-\int_{0}^{T} d t R^{2} S(R) \int_{S^{2}}\left(\bar{\Psi} \gamma^{r} \Psi\right)(t, R) \\
& -\left.\int_{2 r}^{2 R} d s s^{2} S^{-1}(s) \int_{S^{2}}\left(\bar{\Psi} \gamma^{r} \Psi\right)(t, r)\right|_{t=0} ^{t=T} .
\end{aligned}
$$


Since the integrand is static, the last term vanishes, and we conclude that the radial flux is independent of the radius,

$$
r^{2} S(r) \int_{S^{2}}\left(\bar{\Psi} \gamma^{r} \Psi\right)(r)=R^{2} S(R) \int_{S^{2}}\left(\bar{\Psi} \gamma^{r} \Psi\right)(R)
$$

Using (53) and (54), we have

$$
r^{2} S(r) \int_{S^{2}}\left(\bar{\Psi} \gamma^{r} \Psi\right)(r)=\int_{S^{2}} \Phi^{*}(r)\left(\begin{array}{cc}
0 & i \\
-i & 0
\end{array}\right) \Phi(r)
$$

The matching condition (48), expressed in terms of $\Phi$ gives

$$
\lim _{r_{1}<r \rightarrow r_{1}}\left(\begin{array}{cc}
1 & i \\
i & -1
\end{array}\right) \Phi=0
$$

Using this, we have from (63),

$$
\begin{aligned}
\lim _{r_{1}<r \rightarrow r_{1}} r^{2} S(r) \int_{S^{2}}\left(\bar{\Psi} \gamma^{r} \Psi\right)(r) & =\lim _{r_{1}<r \rightarrow r_{1}} \int_{S^{2}}\left[\Phi *\left(\begin{array}{cc}
1 & i \\
-i & 1
\end{array}\right) \Phi-|\Phi|^{2}\right] \\
& =\lim _{r_{1}<r \rightarrow r_{1}} \int_{S^{2}}\left[\Phi *\left(\begin{array}{cc}
1 & 0 \\
0 & -1
\end{array}\right)\left(\begin{array}{cc}
1 & i \\
i & -1
\end{array}\right) \Phi-|\Phi|^{2}\right] \\
& =-\lim _{r_{1}<r \rightarrow r_{1}} \int_{S^{2}}|\Phi|^{2} \neq 0,
\end{aligned}
$$

since $\Phi$ is finite and nonzero on the horizon $r_{1}$.

Now we consider the radial flux for large $R$. Since the flux is independent of $R$, we have from the last inequality

$$
0<\lim _{R \rightarrow \infty}\left|R^{2} S(R) \int_{S^{2}}\left(\bar{\Psi} \gamma^{r} \Psi\right)(R)\right|=\lim _{R \rightarrow \infty}\left|R^{2} S^{-1}(R) \int_{S^{2}}\left(\bar{\Psi} \gamma^{t} \Psi\right)(R)\right|,
$$

because the metric is asymptotically Minkowskian. Thus the integrand of our normalization integral

$$
(\Psi \mid \Psi)_{\infty}=\int_{2 r_{1}}^{\infty} d R R^{2} S^{-1}(R) \int_{S^{2}}\left(\bar{\Psi} \gamma^{t} \Psi\right)(R)
$$

converges to a positive number, so that the normalization integral is infinite.

We have thus proven Part (i) of Theorem 5.1. For Part (ii), the case of an extreme RN background field, we use a quite different method; cf. Ref. 30 .

We remark that, using Chandrasekhar's separation method, the results in this section can be extended to the axisymmetric case. Namely, for a quite general class of axisymmetric black-hole geometries, including the nonextreme Kerr-Newman solution, it is proven in Ref. 33 that the Dirac equation admits no normalizable, time-periodic solutions.

\section{ACKNOWLEDGMENTS}

J.S. research supported in part by the NSF, Grant No. DMS-G-9802370. S-T.Y. research supported in part by the NSF, Grant No. 33-585-7510-2-30. 
${ }^{1}$ D. Christodoulou, "The structure and uniqueness of generalized solutions of the spherically symmetric Einstein-scalar equations," Commun. Math. Phys. 109, 591-611 (1987).

${ }^{2}$ D. Christodoulou, "A mathematical theory of gravitational collapse," Commun. Math. Phys. 109, 613-647 (1987).

${ }^{3}$ D. Christodoulou, "Examples of naked singularity formation in the gravitational collapse of a scalar field," Ann. Math. 140, 607-653 (1994).

${ }^{4}$ D. Christodoulou, "The formation of black holes and singularities in spherically symmetric gravitational collapse," Commun. Pure Appl. Math. 44, 339-373 (1991).

${ }^{5}$ Choptuik, "Universality and scaling in gravitational collapse of a massless scalar field," Phys. Rev. Lett. 70, 9-12 (1993).

${ }^{6}$ R. Bartnik, “Quasispherical metrics and prescribed scalar curvature,” J. Diff. Geom. 37, 31-71 (1993).

${ }^{7}$ R. Bartnik, "Einstein equations in the null quasi-spherical gauge III: Numerical algorithms," gr-qc/9904045.

${ }^{8}$ R. Arnowitt, S. Deser, and C. W. Misner, "The dynamics of general relativity," in Gravitation: An Introduction to Current Research, edited by L. Witten (Wiley, New York, 1962).

${ }^{9}$ R. Schoen and S.-T. Yau, "On the proof of the positive mass conjecture in general relativity," Commun. Math. Phys. 65, 45-76 (1976); ibid. 79, 231-260 (1981).

${ }^{10}$ E. Witten, "A new proof of the positive energy theorem," Commun. Math. Phys. 80, 381-402 (1981).

${ }^{11}$ D. Christodoulou and S. Klainerman, The Global Nonlinear Stability of the Minkowski Space (Princeton University Press, Princeton, 1993).

${ }^{12}$ G. Huisken and T. Ilmanen, "The inverse mean curvature flow and the Riemannian Penrose inequality," J. Diff. Geom. (to be published).

${ }^{13}$ H. Bray, "Proof of the Riemannian Penrose conjecture using the positive mass theorem," (in preparation).

${ }^{14} \mathrm{H}$. Bray and F. Finster, "Curvature estimates and the positive mass theorem," math.DG/9906047.

${ }^{15}$ A. Ashtekar, "A unified treatment of null and spatial infinity in general relativity I. Universal structure, asymptotic symmetries, and conserved quantities at spatial infinity," J. Math. Phys. 19, 1542-1566 (1978).

${ }^{16}$ G. Huisken and S.-T. Yau, "Definition of the center of mass for isolated physical systems and unique foliations by stable spheres with constant mean curvature,"' Invent. Math. 124, 281-311 (1996).

${ }^{17}$ D. Christodoulou and S.-T. Yau, "Some remarks on the quasilocal mass," Am. Math. Soc., Prov. RI, Series: Contemp. Math. 71, 9-14 (1988).

${ }^{18}$ R. Schoen and S.-T. Yau, "The existence of a black hole due to condensation of matter," Commun. Math. Phys. 90, 575-579 (1983).

${ }^{19}$ R. Adler, M. Bazin, and M. Schiffer, Introduction to General Relativity (McGraw-Hill, New York, 1975).

${ }^{20}$ R. Bartnik and J. McKinnon, "Particlelike solutions of the Einstein-Yang-Mills equations,"' Phys. Rev. Lett. 61, 141-144 (1988).

${ }^{21}$ H. P. Kunzle and A. K. M. Masood-ul-Alam, "Spherically symmetric static SU(2) Einstein-Yang-Mills fields," J. Math. Phys. 31, 928-935 (1990).

${ }^{22}$ J. Smoller and A. Wasserman, "Existence of infinitely many smooth, static global solutions of the Einstein-Yang/Mills equations," Commun. Math. Phys. 151, 303-325 (1993).

${ }^{23}$ S. Coleman, in New Phenomena in Subnuclear Physics, edited by A. Zichichi (Plenum, New York, 1975).

${ }^{24}$ S. Deser, “Absence of static solutions in source-free Yang-Mills theory," Phys. Lett. B 64, 463-465 (1976).

${ }^{25}$ N. Straumann and Z. Zhou, “Instability of the Bartnik-McKinnon solution,"' Phys. Lett. B 237, 353-356 (1990).

${ }^{26}$ T. D. Lee, “Mini-soliton stars,” Phys. Rev. D 25, 3640-3657 (1987).

${ }^{27}$ F. Finster, J. Smoller, and S.-T. Yau, "Particlelike solutions of the Einstein-Dirac equations," gr-qc/9801079, Phys. Rev. D 59, 104020 (1999).

${ }^{28}$ F. Finster, J. Smoller, and S.-T. Yau, “Particlelike solutions of the Einstein-Dirac-Maxwell equations,” gr-qc/9802012, Phys. Lett. A 259, 431-436 (1999).

${ }^{29}$ F. Finster, J. Smoller, and S.-T. Yau, "Nonexistence of black hole solutions for a spherically symmetric Einstein-DiracMaxwell system,”' gr-qc/9810048, Commun. Math. Phys. 205, 249-262 (1999).

${ }^{30}$ F. Finster, J. Smoller, and S.-T. Yau, "Nonexistence of time-periodic solutions of the Dirac equation in a ReissnerNordström black hole background," gr-qc/9805050, J. Math. Phys. (to be published).

${ }^{31}$ F. Finster, J. Smoller, and S.-T. Yau, "The coupling of gravity to spin and electromagnetism," Mod. Phys. Lett. A 14, 1053-1057 (1999).

${ }^{32}$ J. Smoller, A. Wasserman, and S.-T. Yau, "Existence of black-hole solutions for the Einstein-Yang/Mills equations," Commun. Math. Phys. 154, 377-401 (1993).

${ }^{33}$ F. Finster, N. Kamran, J. Smoller, and S.-T. Yau, "Nonexistence of time-periodic solutions of the Dirac equation in an axisymmetric black hole geometry," gr-qc/9905047.

${ }^{34}$ J. J. Sakurai, Advanced Quantum Mechanics (Addison-Wesley, Reading, 1967).

${ }^{35}$ J. Smoller, Shock Waves and Reaction-Diffusion Equations, 2nd ed. (Springer-Verlag, Berlin, 1994).

${ }^{36}$ S. W. Hawking, "Particle creation by black holes," Commun. Math. Phys. 43, 199-220 (1975). 